\title{
A Comparative Study and Development of a Passive Robot with Improved Stability
}

\author{
Hamid Reza Shafei ${ }^{1}$, Soroush Sadeghnejad ${ }^{2(\bowtie)}$, \\ Mohsen Bahrami ${ }^{1}$, and Jacky Baltes ${ }^{3}$ \\ ${ }^{1}$ Mechanical Engineering Department, \\ Amirkabir University of Technology (Tehran Polytechnic), Tehran, Iran \\ \{hr.shafei,mbahrami\}@aut.ac.ir \\ ${ }^{2}$ Amirkabir Robotic Institute, \\ Amirkabir University of Technology (Tehran Polytechnic), Tehran, Iran \\ s.sadeghnejad@aut.ac.ir \\ 3 Autonomous Agents Laboratory, University of Manitoba, Winnipeg, Canada \\ jacky@cs.umanitoba.ca
}

\begin{abstract}
Passive walkers are robots that can produce a stable cyclical movement similar to walking on mildly inclined surfaces. In recent years, various investigations have been conducted on this subject. Since this is a new field of research, the effect of structural parameters on the movement of these walkers and the development of more human-like movement models can be further investigated. This paper compares three popular models for passive dynamic walkers: Garcia's mode, Wisse's model, and our own extended Wisse's model with arm. Our research shows that the extended models lead to more energy efficient and stable walking.
\end{abstract}

\section{Introduction}

Among the mobile robots, the bipedal robots have always been of interest, because of their adequate flexibility and movement speed; however, the high energy consumption of these robots has made their use impractical. Since the presentation of the first passive walker model by McGeer in 1990, a new window was opened to the investigation of the walking process in humans and to the designing of highly-efficient robots [4]. Through the passive walking mechanism using the two feet, humans are able to walk on inclined surfaces without needing any control, and by spending only a small amount of input energy. In Fig. 1, the energy utilization of most famous active and passive bipedal robots and of humans has been compared.

Walking appears as a very complicated process, which is controlled by the muscular and nervous systems. But, is this control system fully active, or can it operate passively in certain conditions? By definition, a mechanical system is said to have a passive movement, if, during the movement, the resultant of the input gravitational energy and the energy losses in it is zero and no outside energy and control are applied to the system. This notion was first introduced by McMohan et al. [3].

They maintained that the oscillatory motion of human feet can be convincingly modeled as a passive dual pendulum. They called this type of movement "Throw 


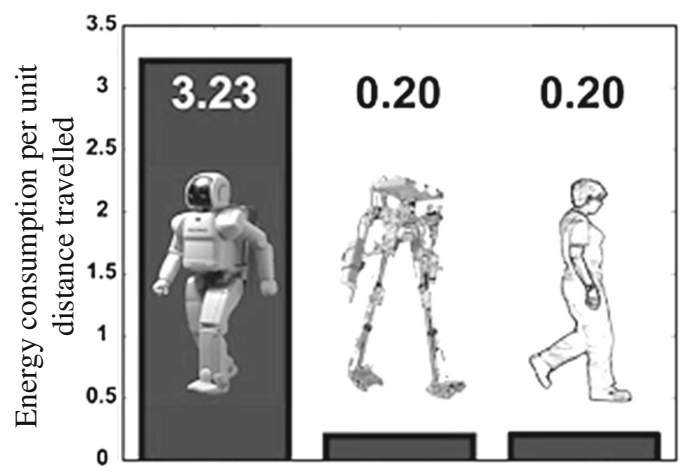

Fig. 1. Comparison of human energy, Honda's robot and semi-active biped robot at Cornell University [2]

Walking". Later on, in 1990, McGeer demonstrated that walking can be a completely autonomous [self-initiated] process. He stated: "A set of bi-pedal machines exist for which, walking is a natural dynamic mode. The movement started on a mildly inclined surface by a machine belonging to this set leads to a stable movement comparable to natural walking, while it requires no input energy and control." [4] This stable movement by two feet is sustained by balancing the energy lost during the impact of feet with the ground and the other plausible energy losses during movement with the input energy due to gravity. McGeer demonstrated this fact theoretically as well as experimentally by building prototypes of bipedal walkers. This line of research was subsequently pursued by other investigators who presented different models. These models included the 3D models [5], models with upper bodies [6], and models with knees [7]. The most complex passive bipedal walker is a 3D model with knees and arms, which was built in the Cornell University under the supervision of Andy Ruina in 2001 (Fig. 2) [5]. In recent years, many universities around the world have conducted research works on passive and semi-active bipedal walkers [8-13]. In brief, the advantages of the passive movement scheme are as follows: (1) Saving in the consumption of energy: while the humanoid robot of the Honda Motors Company carries a $6 \mathrm{~kg}$ battery for $40 \mathrm{~min}$ of walking [1], Garcia et al. demonstrated that the energy utilization limit of a passive bipedal walker can be near zero, (2) the natural movement modes of passive walkers are very similar to human walking modes.

In 1988, Garcia designed the simplest bipedal robot model that could not be reduced further [1]. Garcia's model consisted of 2 massless rods, with 3 point masses at the ends of two feet and the thigh joint; and the whole model moved two-dimensionally (Fig. 1). He showed that the above mechanism can walk on mildly inclined surfaces (Fig. 3).

In 2004, the team of Wisse and Schwab of the Delft University analyzed a bipedal robot similar to Garcia's, but which had an upper body [6]. The upper body had a kinematic constraint that always kept it aligned with the bisector of the angle formed between the two feet (Fig. 4). The effect of the upper body on stability was analyzed and it was demonstrated that the existence of the upper body increases the walking speed and efficiency in the robot. Finally, it was shown that by adding a spring to the 


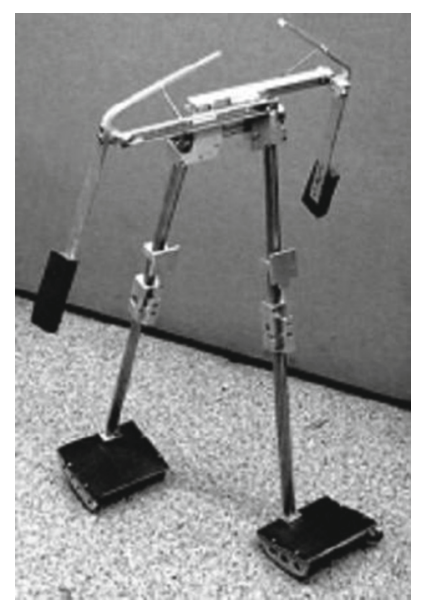

Fig. 2. 3D passive walking at Cornell University [7]

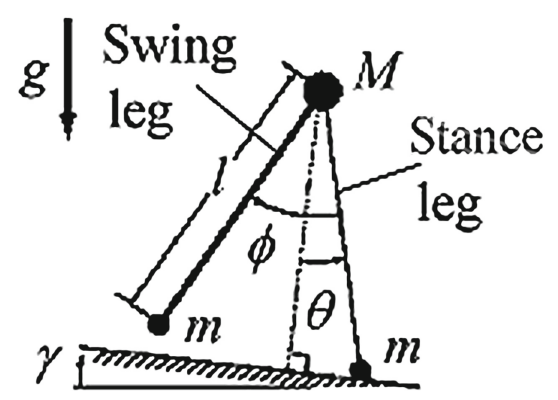

Fig. 3. Garcia's model [1]

above model, the stride length can be arbitrarily changed. In this paper, Wisse's robot was modeled by placing a spring at the connection joint of the two feet and analyzed the speed and robustness of the resulting walking gait. This robot is shown in Fig. 4.

In our research, we extended the previous models by Garcia and Wisse to create a more realistic and accurate model with springs and arms for a walker robot and analyzed its behavior with respect to walking speed and stability. The model is based on the same prototype as presented by Wisse and Schwab, except that instead of using a kinematic constraint to hold the upper body upright, a torsional spring is introduced (Fig. 5).

\section{Modeling of Garcia's Robot}

Due to the discretely continuous responses of the system, two sets of equations are needed to describe the overall motion. The Euler-Lagrange relations and the Hamilton principle are used to obtain the equations of motion governing the movement of the bipedal robot. First, the potential and kinetic energy equations are formulated, and then 


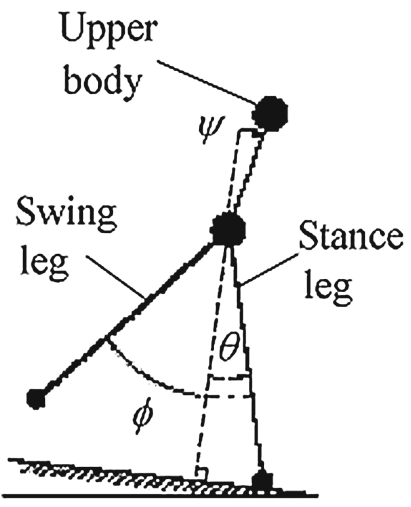

Fig. 4. Wisse's original model [12]

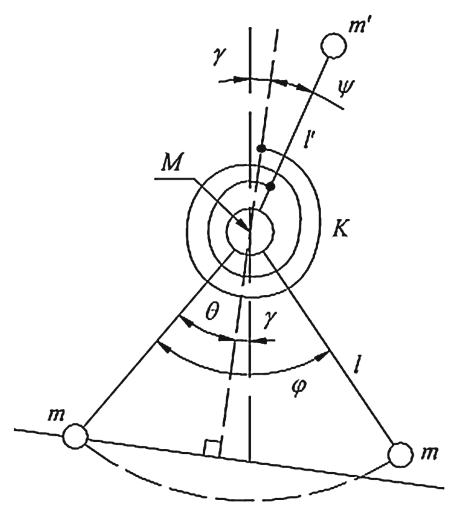

Fig. 5. Extended Wisse's model [9]

the equations of motions are derived by taking the derivative of the energy functions. The equations obtained at this stage, accurately describe the movement of the two feet at all times with the exception of the time when the feet impact on the ground. The general form of the equations is expressed as relation (1).

$$
M(q) \ddot{q}+C(q, \dot{q}) \dot{q}+g(q)=0
$$

Here, $q$ is the state vector and is written as $\{\theta, \phi\}^{T}$ representing the stance and swing leg angles respectively. This way, two equations of motion are obtained. Since many parameters exist in the equations, and non-dimensionalizing the equations can facilitate the evaluation of the responses and also the comparison with other models, the following non-dimensionalizing variable expressing the ratio of foot to hip mass is used:

$$
\beta=\frac{m}{M}
$$

Thus, by changing the above variable, the equations of motions are obtained as follows [1]:

$$
\left\{\begin{array}{l}
{[1+2 \beta(1-\cos \varphi)] \ddot{\theta}-\beta(1-\cos \varphi) \ddot{\varphi}} \\
-\beta(\dot{\varphi}-2 \dot{\theta}) \dot{\varphi} \sin \varphi+\frac{\beta g}{l}[\sin (\theta-\varphi-\psi)-\sin (\theta-\psi)] \\
-\frac{g}{l} \sin (\theta-\psi)=0 \\
\ddot{\varphi}-(1-\cos \varphi) \ddot{\theta}-\dot{\theta}^{2} \sin \varphi-\frac{g}{l} \sin (\theta-\varphi-\psi)=0
\end{array}\right.
$$

Now, by assuming $m<<M, \beta=0$ (i.e., the mass of feet is ignored with respect to the mass of the waist), the equations take the following form [1]: 


$$
\left\{\begin{array}{l}
\ddot{\theta}-\sin (\theta-\gamma) \frac{g}{l}=0 \\
-\ddot{\varphi}+\ddot{\theta}+\dot{\theta}^{2} \sin \varphi-\cos (\theta-\gamma) \sin \varphi=0
\end{array}\right.
$$

The impact condition for this robot is defined as follows:

$$
\varphi=-2 \theta
$$

It should be mentioned that the above condition also applies when both angles are equal to zero; however, this situation is ignored in the analysis and the assumption is that the swing leg can swing freely through this condition.

By using the conservation of angular momentum about the stance point and impact point, the transfer matrix is obtained as:

$$
\left[\begin{array}{c}
\theta \\
\dot{\theta} \\
\varphi \\
\dot{\varphi}
\end{array}\right]^{+}=\left[\begin{array}{cccc}
-1 & 0 & 0 & 0 \\
0 & \cos (2 \theta) & 0 & 0 \\
-2 & 0 & 0 & 0 \\
0 & \cos (2 \theta)(1-\cos (2 \theta)) & 0 & 0
\end{array}\right]\left[\begin{array}{c}
\theta \\
\dot{\theta} \\
\varphi \\
\dot{\varphi}
\end{array}\right]^{-}
$$

We simulated the resulting system using Matlab's builtin Runge Kutta solver for differential equations.

The initial conditions and the parameters are shown in Table 1.

Table 1. Parameters and Initial conditions for Garcia's model [1]

\begin{tabular}{l|r|l|l}
\hline \multicolumn{2}{l|}{ Initial conditions } & \multicolumn{2}{l}{ Parameters } \\
\hline$\theta$ & 0.2073 & $\gamma$ & 0.009 \\
\hline$\dot{\theta}$ & -0.2061 & & \\
\hline$\varphi$ & 0.4147 & & \\
\hline$\dot{\varphi}$ & -0.0175 & & \\
\hline
\end{tabular}

The results of the simulations are shown in Fig. 6.

As the above figure shows, the limit cycle is obviously stable. It should be mentioned that the above limit cycle has become stable at the slope angle less than 0.009 .

\section{Modeling of Wisse's Robot}

The dynamic equations of Wisse's robot are obtained by employing the Lagrange formulation. The general form of these equations is expressed by relation (1), but the vector of state variables is extended by adding a third dimension - the hip angle

$$
q=\theta, \varphi, \psi^{T}
$$




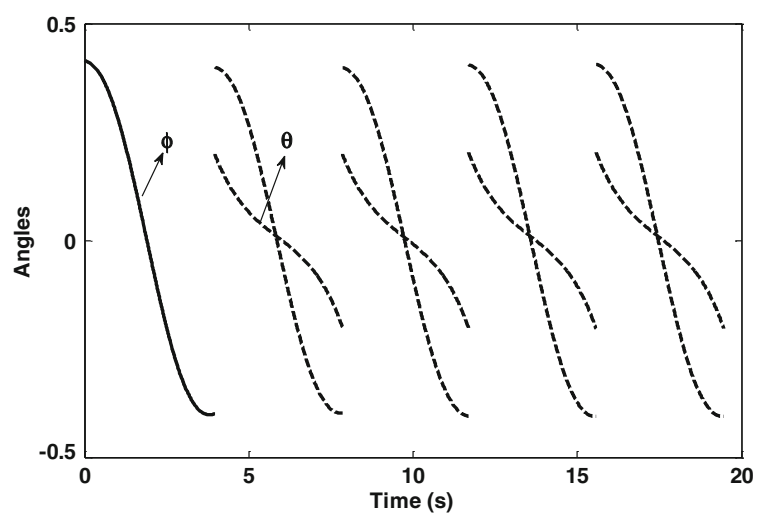

Fig. 6. Stable limit cycle, for slope angles $0.009 \mathrm{Rad}$

Consequently, three equations of motion are generated, and according to the previously mentioned explanations, these equations are converted to non-dimensionalized forms. So, the dimensionless parameters are defined as follows:

$$
\alpha=\frac{m^{\prime}}{M}, \quad \beta=\frac{m}{M}, \quad k=\frac{K}{M \lg }, \quad v=\frac{l^{\prime}}{l}
$$

Therefore, the following dimensionless equations are obtained [9]:

$$
\begin{aligned}
& {\left[M l^{2}+2 m l^{2}(1-\cos \varphi)+m^{\prime} l^{2}\right] \ddot{\theta}} \\
& -m l^{2}(1-\cos \varphi) \ddot{\varphi}-m^{\prime} l^{2} v \cos (\psi+\theta) \ddot{\psi} \\
& -M l^{2} \dot{\varphi}^{2} \sin \varphi+2 M l^{2} \dot{\varphi} \dot{\theta} \sin \varphi+m^{\prime} l^{2} v \dot{\psi}^{2} \sin (\psi+\theta) \\
& -M g l \sin (\theta-\gamma)-m^{\prime} g l \sin (\theta-\gamma) \\
& -M g l[\sin (\theta-\gamma)-\sin (\varphi-\theta-\gamma)]=0 \\
& m l^{2}(1-\cos \varphi) \ddot{\theta}-m l^{2} \ddot{\varphi}+m l^{2} \dot{\theta}^{2} \sin \phi \\
& +m g l \sin (\theta-\varphi-\psi)=0 \\
& -m^{\prime} l^{2} v \cos (\theta+\psi) \ddot{\theta}+m^{\prime} l^{2} v^{2} \ddot{\psi} \\
& +m^{\prime} l^{2} v \dot{\theta}^{2} \sin (\theta+\psi)-m^{\prime} g l^{\prime} \sin (\psi-\gamma)+k \psi=\tau
\end{aligned}
$$

Similarly, the impact condition for this robot is defined as:

$$
\varphi=-2 \theta
$$

The transfer matrix for the Wisse's robot can be obtained by writing the conservation of angular momentum about the following three points: 
About the hip, for the left foot.

About the hip, for the upper body.

About the flight foot, for the whole body.

$$
\left[\begin{array}{c}
\theta \\
\dot{\theta} \\
\varphi \\
\dot{\varphi} \\
\psi \\
\dot{\psi}
\end{array}\right]^{+}=\left[\begin{array}{cccccc}
-1 & 0 & 0 & 0 & 0 & 0 \\
0 & A & 0 & 0 & 0 & 0 \\
2 & 0 & 0 & 0 & 0 & 0 \\
0 & B & 0 & 0 & 0 & 0 \\
0 & 0 & 0 & 0 & 1 & 0 \\
0 & C & 0 & 0 & 0 & 1
\end{array}\right]\left[\begin{array}{c}
\theta \\
\dot{\theta} \\
\varphi \\
\dot{\varphi} \\
\psi \\
\dot{\psi}
\end{array}\right]{ }^{-}
$$

Where we have:

$$
\begin{aligned}
& A=\frac{\mu(\cos (2 \theta)-\cos (2 \psi))+2 \cos (2 \theta)}{2 \beta \sin ^{2}(2 \theta)+2 \mu \sin ^{2}(\theta-\psi)+2} \\
& B=A(1-\cos (2 \theta)) \\
& C=\left[1+\frac{\cos (\theta-\psi)}{v}\right](1-A)
\end{aligned}
$$

By performing the simulation, the limit cycle of the robot is obtained as follows (Table 2):

Table 2. Parameters and Initial conditions for Wisse's model [9]

\begin{tabular}{l|l|l|l}
\hline \multicolumn{2}{l|}{ Initial conditions } & \multicolumn{2}{l}{ Parameters } \\
\hline$\theta$ & 0.2 & $\gamma$ & 0.0045 \\
\hline$\dot{\theta}$ & -0.2 & $\beta$ & 0 \\
\hline$\varphi$ & 0.4 & $\alpha$ & 0.08 \\
\hline$\dot{\varphi}$ & -0.02 & $v$ & 0.13 \\
\hline$\psi$ & -0.3659 & $k$ & 0.0917 \\
$\dot{\psi}$ & 1.7925 & & \\
\hline
\end{tabular}

And the results are as follows (Fig. 7):

The above figure clearly shows the stability of the limit cycle. The limit cycle is stable with a slope angle less than 0.0045 .

\section{Modeling of a Passive Robot with Arms}

In this section, a 5-DOF robot consisting of two arms, two feet and also one degree of freedom in the trunk section is analyzed. The configuration of this robot has made it more human-like. This robot is shown in Fig. 8. 


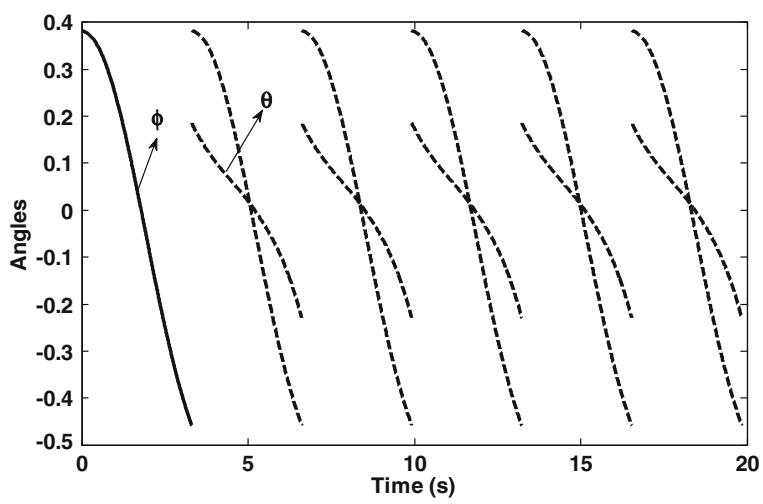

Fig. 7. Stable limit cycle, for slope angles $0.0045 \mathrm{Rad}$

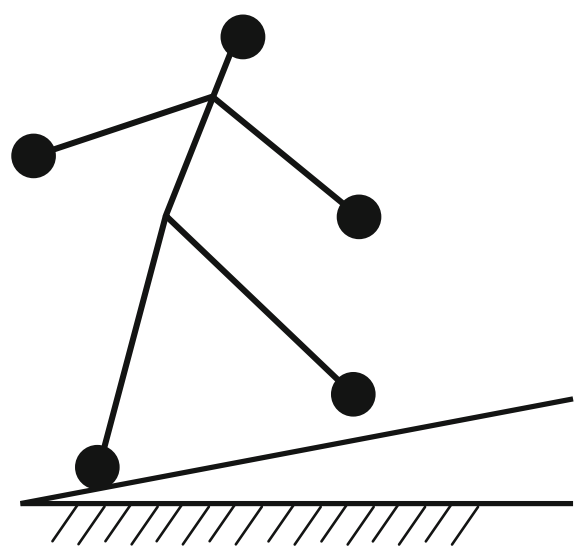

Fig. 8. 5 DOF model

Similar to the previous robot, Lagrange formulation is used to extract the dynamic equations of this system. The kinetic energy of this system is written as:

$$
\begin{aligned}
T_{1} & =\frac{1}{2} m_{1} l_{1}^{2} \dot{\theta}_{1}^{2} \\
T_{2} & =\frac{1}{2} m_{2} l_{1}^{2}\left(\dot{\theta}_{1}^{2}+\left(\dot{\theta}_{1}-\dot{\varphi}\right)^{2}-2 \dot{\theta}_{1}\left(\dot{\theta}_{1}-\dot{\varphi}\right) \cos \varphi\right) \\
T_{3} & =\frac{1}{2} m_{3}\left(l_{1}^{2} \dot{\theta}_{1}^{2}+l_{2}^{2}\left(\dot{\theta}_{1}+\dot{\theta}_{2}\right)^{2}-2 l_{1} l_{2} \dot{\theta}_{1}\left(\dot{\theta}_{1}+\dot{\theta}_{2}\right) \cos \left(\theta_{2}-\theta_{1}\right)\right) \\
T_{4} & =\frac{1}{2} m_{4}\left(l_{1}^{2} \dot{\theta}_{1}^{2}+l_{2}^{2}\left(\dot{\theta}_{1}-\dot{\theta}_{3}\right)^{2}-2 l_{1} l_{2} \dot{\theta}_{1}\left(\dot{\theta}_{1}-\dot{\theta}_{3}\right) \cos \left(\theta_{1}-\theta_{3}\right)\right) \\
T_{5} & =\frac{1}{2} m_{5}\left(l_{1}^{2} \dot{\theta}_{1}^{2}+\left(\dot{\theta}_{1}-\dot{\psi}\right)^{2} l_{3}^{2}+2 l_{1} l_{3} \dot{\theta}_{1}\left(\dot{\theta}_{1}-\dot{\psi}\right) \cos \left(\theta_{1}-\psi\right)\right)
\end{aligned}
$$


And potential energy:

$$
\begin{aligned}
& V_{1}=m_{1} g l_{1} \cos \left(\theta_{1}-\gamma\right) \\
& V_{2}=m_{2} g l_{1}\left(\cos \left(\theta_{1}-\gamma\right)-\cos \left(\theta_{1}-\varphi-\gamma\right)\right) \\
& V_{3}=m_{3} g\left(l_{1} \cos \left(\theta_{1}-\gamma\right)-l_{2} \cos \left(\theta_{2}-\gamma\right)\right) \\
& V_{4}=m_{4} g\left(l_{1} \cos \left(\theta_{1}-\gamma\right)-l_{2} \cos \left(\theta_{3}+\gamma\right)\right) \\
& V=m_{5} g\left(l_{1} \cos \left(\theta_{1}-\gamma\right)+l_{3} \cos (\psi+\gamma)\right)+\frac{1}{2} k \psi^{2}
\end{aligned}
$$

By inserting the kinetic and potential energies into the Lagrange relation, the dynamic equations of the above system are obtained, employing the previously mentioned impact conditions and using the initial conditions and the dimensionless parameters which are defined below (Table 3):

Table 3. Parameters and Initial conditions for 5 DOF's model

\begin{tabular}{l|l|l|l}
\hline \multicolumn{2}{l|}{ Initial conditions } & \multicolumn{2}{l}{ Parameters } \\
\hline$\theta$ & 0.2 & $\gamma$ & 0.01 \\
\hline$\dot{\theta}$ & -0.2 & $\beta$ & 0.06 \\
\hline$\varphi$ & 0.38 & $\alpha$ & 0 \\
\hline$\dot{\varphi}$ & -0.02 & $v$ & 0.2 \\
\hline$\psi$ & -0.3659 & $k$ & 0.0917 \\
\hline$\dot{\psi}$ & 1.7925 & $\xi$ & 0.07 \\
\cline { 2 - 4 } & & $\sigma$ & 0.004 \\
\hline
\end{tabular}

$$
\alpha=\frac{m_{2}}{m_{1}}, \quad \beta=\frac{m_{3}=m_{4}}{m_{1}}, \quad \sigma=\frac{m_{5}}{m_{1}}, \quad \frac{l_{2}}{l_{1}}=v, \quad \frac{l_{3}}{l_{1}}=\xi
$$

By performing the simulations, the following results are obtained (Figs. 9 and 10):

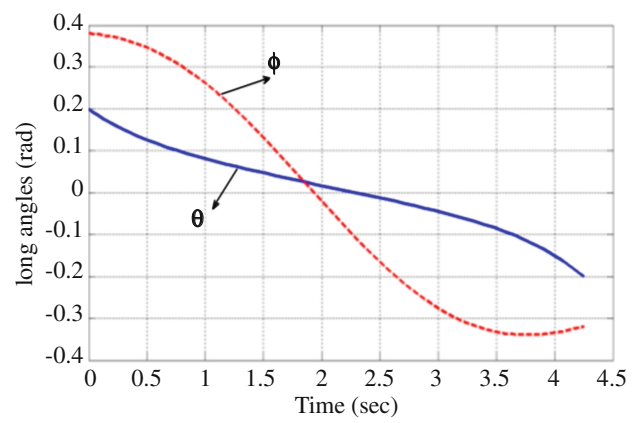

Fig. 9. Stable limit cycle, for slope angle $0.01 \mathrm{Rad}$ 


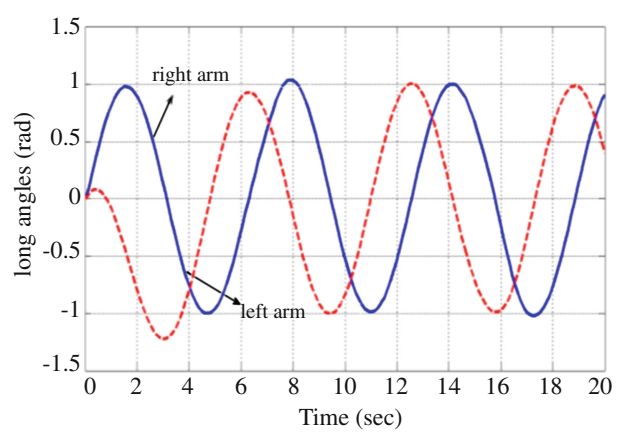

Fig. 10. The position of the robot's arm

The reason for changing the sign of angles $\theta$ and $\varphi$ is the change in the measuring reference of these angles at the moment of impact. Because, as the support (fulcrum) foot suddenly switches, the two said angles find new definitions and a new measuring reference, and therefore, from a geometrical perspective, their signs will change. But since the measuring reference of angle $\psi$ doesn't change during impact, this angle always remains the same; because, although the impact forces produce impulsive changes in acceleration and stepped changes in velocity, they are not able to change the angles themselves. So, if the measuring references of the angles are fixed, these angles will always remain constant. At this stage, the 5-DOF robot has attained a stable limit cycle at a slope less than 0.01 .

\section{Conclusion}

In this paper, three models of passive robots were analyzed. For each system, a stable limit cycle was achieved by finding appropriate initial conditions and dimensionless parameters. In the Garcia's model, the robot attained a stable limit cycle at slope angle less than 0.009; whereas in the Wisse's model and the model with arms, the robots achieved stability at slopes angle less than 0.0045 and 0.01 , respectively. Therefore, we can conclude that by extending the Wisse's robot model to a 5-DOF robot with arms, the new robot becomes more stable.

\section{References}

1. Garcia, M., Chatterjee, A., et al.: The simplest walking model stability, complexity, and scaling. J. Biomech. Eng. 120(2), 281-288 (1998)

2. http://www-personal.umich.edu/ shc/Robot/

3. McMahon, T., Rose, J., and Gamble, J.G.: Human Walking, Biomechanical engineering, pp. 52-53, (1989)

4. McGeer, T: Passive Dynamic Walking, School of Engineering Science Simon Fraser University, May 1988 
5. Kuo, A.D.: Stabilization of lateral motion in passive dynamic walking. Int. J. Robot. Res. 18 (9), 917-930 (1999)

6. Wisse, M., Schwab, A.L.: Passive dynamic walking model with upper body. Robotica 22, 681-688 (2004). Cambridge University Press

7. Collins, S., Wisse, M., Ruina, A.: A three-dimensional passive dynamic walking robot with two legs and knees. Int. J. Robot. Res. 20, 607-622 (2001)

8. Collins S., Ruina A., A bipedal walking robot with efficient and human-like gait. In: International Conference on Robotics and Automation - ICRA, pp.1983-1988, Barcelona, Spain (2005)

9. Farshimi, F., Naraghi, M.: A passive-biped model with multiple routes to chaos. J. Acta Mech. Sinica 27(2), 277-284 (2011)

10. Safa, A.T., Saadat, M.G., Naraghi, M.: Passive dynamic of the simplest walking model replacing ramps with stairs. Mech. Mach. Theory 42, 1314-1325 (2007). Elsevier Publication

11. Camp J.: Powered "Passive" dynamic walking, Masters of Engineering Project, The Sibley School of Mechanical and Aerospace Engineering, Cornell University, USA, pp. 1-8 (1997)

12. Wisse M.: Essentials of dynamic walking, analysis and design of two-legged robots, Delft University, Netherlands, pp. 33-50 (2004). ISBN 90-77595-82-1

13. Tedrake R., Zhang W., et al., Actuating a Simple3D passive dynamic walker. In: IEEE International Conference on Robotics and Automation, New Orleans, USA, pp. 1-6 (2004) 